\title{
Kumaraswamy Inverse Flexible Weibull Distribution: Theory and Application
}

\author{
Jamal N. Al Abbasi \\ Al Nahrain University \\ Baghdad - IRAQ
}

\begin{abstract}
A generalization of the Inverse flexible weibull distribution so-called the Kumaraswamy-Inverse flexible weibull distribution is proposed and studied. Various structural properties including explicit expressions for the moments, quantiles and moment generating function of the new distribution are derived. The estimation of the model parameters is performed by maximum likelihood method and the observed Fisher's information matrix is derived. For different values of sample sizes, Monte Carlo simulation is performed to investigate the precision of the maximum likelihood estimates. The usefulness of the kumaraswamy inverse flexible distribution for modeling data is illustrated using real data.
\end{abstract}

\section{Keywords}

Flexible Weibull Distribution; Kumaraswamy-G Class; Hazard Function; Maximum Likelihood; Reliability.

\section{INTRODUCTION}

The Inverse Flexible Weibull distribution was introduced by El-Gohary et.al. [1], and it has been studied and discussed as a lifetime model. If a random variable $\mathrm{Y}$ has a flexible weibull extension FW distribution [2], the variable $\mathrm{X}=\frac{1}{\mathrm{Y}}$ will have an Inverse Flexible Weibull Extension IFW distribution. Thus, a random variable $\mathrm{X}$ is said to have an Inverse Flexible Weibull Extension distribution with parameters $\mu>0$ and $\sigma>0$ if it's cumulative distribution function (cdf) are given by;

$$
\mathrm{F}(\mathrm{x} ; \mu, \sigma)=\mathrm{e}^{-\mathrm{e}^{\frac{\mu}{x}-\sigma x}}, \mathrm{x}>0, \mu, \sigma>0
$$

The probability density function (pdf) corresponding to (1) becomes

$$
\mathrm{f}(\mathrm{x} ; \mu, \sigma)=\left\{\frac{\mu}{\mathrm{x}^{2}}+\sigma\right\} \mathrm{e}^{\frac{\mu}{\mathrm{x}}-\sigma \mathrm{x}} \mathrm{e}^{-\mathrm{e}^{\frac{\mu}{\mathrm{x}}-\sigma \mathrm{x}}}, \mathrm{x}>0, \mu, \sigma>0
$$

In many practical situations, classical distributions do not provide adequate fit to real data. So, several generators of introducing one or more parameters to generate new distributions have been studied recently in the statistical literature. Some well-known generators are Marshall-Olkin generated family (MO-G) by Marshall and Olkin [3], beta $G$ by Eugene et al. [4], Transmuted $-G$ by Shaw and Buckley [5], Kumaraswamy-G (K-G) by Cordeiro and de Castro [6], McDonald-G (Mc-G) by Alexander et al. [7], gamma-G (type 1) by Zografos and Balakrishanan [8], gamma-G (type 2) by Risti'c and Balakrishanan [9], loggamma-G by Amini et al. [10], logistic-G by Torabi and Montazari [11], exponentiated generalized-G by Cordeiro et al [12], Transformed-Transformer (T-X) by Alzaatreh et al. [13], Weibull-G by Bourguignon et al. [14], and logistic-X by Tahir et al.[15] . Among these generators, the K-G family has received increased attention after the convincing debate on the pitfalls of the beta-G family by Jones [16]. For a baseline random variable having pdf $\mathrm{g}(\mathrm{x})$ and $\mathrm{cdf} \mathrm{G}(\mathrm{x})$, Cordeiro and de Castro [6] defined the two-parameter K-G cdf by

$$
\mathrm{F}(\mathrm{x})=1-\left\{1-\mathrm{G}(\mathrm{x})^{\alpha}\right\}^{\beta}
$$

The pdf corresponding to (3) becomes

$$
f(x)=\alpha \beta G(x)^{\alpha-1}\left\{1-G(x)^{\alpha}\right\}^{\beta-1}
$$

Where $\mathrm{g}(\mathrm{x})=\frac{\mathrm{dG}(\mathrm{x})}{\mathrm{dx}}$ and $\alpha>0$ and $\beta>0$ are two extra shape parameters whose role are to govern skewness and tail weights.

In this Article, the main propose an extension of the IFW model called the Kumaraswamy Inverse Flexible Weibull Extension KIFW "for short" distribution based on equations (3) and (4). This paper is organized as follows. Section 2, defines the cumulative, density and hazard functions of the KIFW distribution. Following that (Section 3) introduces some mathematical and statistical properties including, quantile function, moments and moment generating function. The maximum likelihood estimation of the parameters is determined in Section 4. Section 5 evaluates the performance of a maximum likelihood method by using Monte Carlo simulation. Real data sets are analyzed in Section 6 and the results are compared with existing distributions. Finally, concluding remarks are addressed in section 7 .

\section{KUMARASWAMY INVERSE}

\section{FLEXIBLE WEIBULL DISTRIBUTION}

Inserting (2) in (4), the four-parameter KIFW cdf is defined by

$$
\mathrm{F}(\mathrm{x})=1-\left[1-\mathrm{e}^{-\alpha \mathrm{e}^{\frac{\mu}{\mathrm{x}}-\sigma \mathrm{x}}}\right]^{\beta}, \mathrm{x}>0, \alpha, \beta, \mu, \sigma>0
$$

The pdf corresponding to (5) becomes

$$
\mathrm{f}(\mathrm{x})=\alpha \beta\left\{\frac{\mu}{\mathrm{x}^{2}}+\sigma\right\} \mathrm{e}^{\frac{\mu}{\mathrm{x}}-\sigma \mathrm{x}} \mathrm{e}^{-\alpha \mathrm{e}^{\frac{\mu}{x}-\sigma x}}\left[1-\mathrm{e}^{-\alpha \mathrm{e}^{\frac{\mu}{x}-\sigma x}}\right]^{\beta-1}
$$

For notational purpose, we write; $\mathrm{X} \sim \operatorname{KIFW}(\alpha, \beta, \mu, \sigma)$ It can be seen that if $\alpha=\beta=1$, the distribution in Equation (6) reduces to an Inverse Flexible Weibull Extension Distribution with parameters $\mu$ and $\sigma$.

Figure 1 illustrates the graphical behavior of the pdf of Kumaraswamy inverse Flexible Weibull distribution for selected values of the parameters $\alpha$ and $\beta$ with $\mu=1.2$ and $\sigma=$ 1.8 .

The survival function for the KIFW distribution is given by;

$$
\mathrm{S}(\mathrm{x})=1-\mathrm{F}(\mathrm{x})=\left[1-\mathrm{e}^{-\alpha \mathrm{e}^{\frac{\mu}{x}-\sigma \mathrm{x}}}\right]^{\beta}, \mathrm{x}>0,
$$

The hazard function is thus given by; 


$$
h(x)=\frac{f(x)}{S(x)}=\frac{\alpha \beta\left\{\frac{\mu}{x^{2}}+\sigma\right\} e^{\frac{\mu}{x}-\sigma x} \mathrm{e}^{-\alpha e^{\frac{\mu}{x}-\sigma x}}}{\left[1-\mathrm{e}^{-\alpha e^{\frac{\mu}{x}-\sigma x}}\right]}
$$
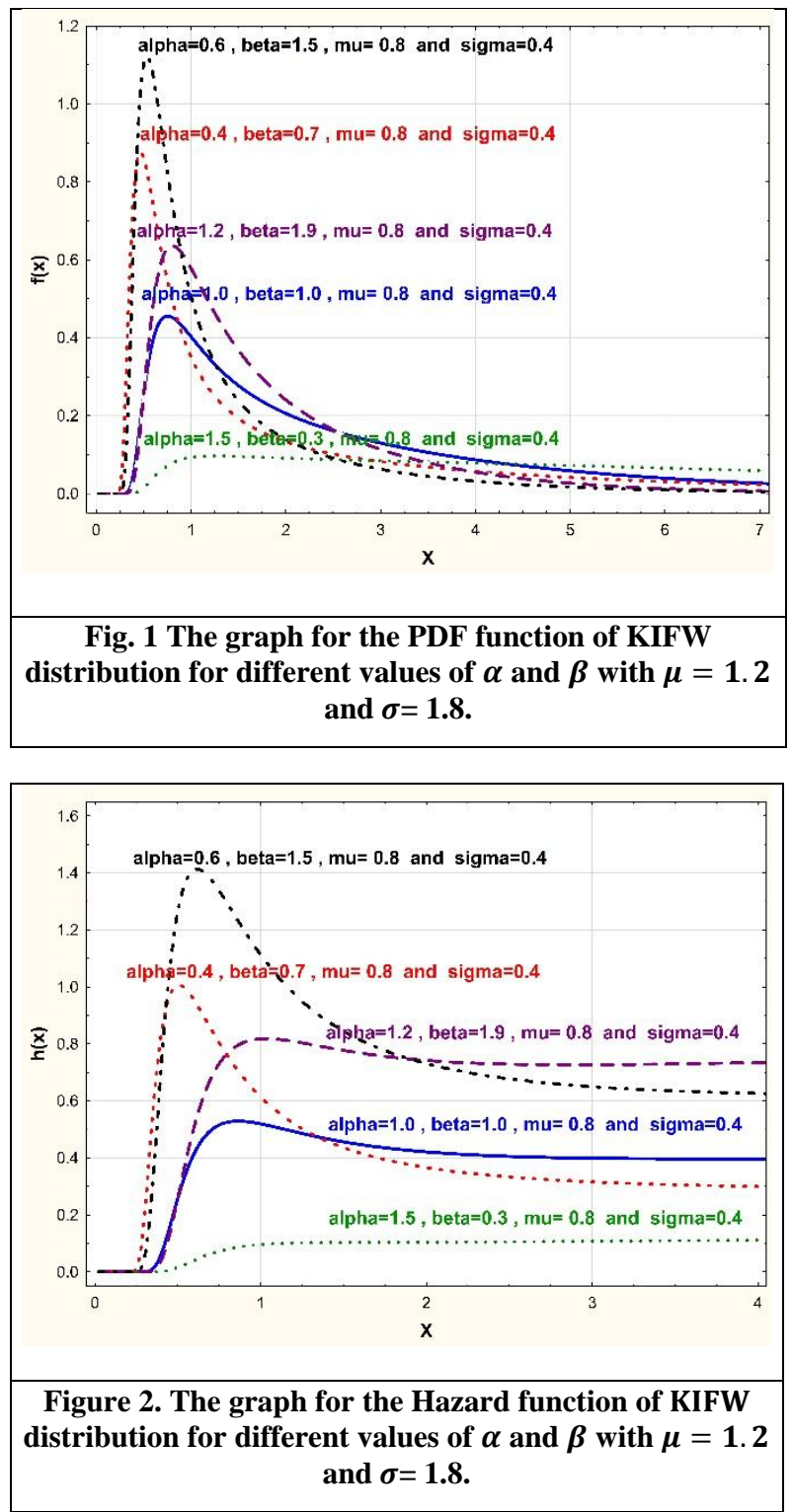

\section{MATHEMATICAL AND} STATISTICAL PROPERTIES

\subsection{Useful Expansions}

By using the generalized binomial theorem, can be used $\left[1-\mathrm{e}^{-\alpha e^{\frac{\mu}{x}-\sigma x}}\right]^{\beta-1}=\alpha \beta \sum_{i=0}^{\infty} \frac{(-1)^{i}}{i !} \frac{\Gamma(\beta)}{\Gamma(\beta-i)} \exp \left[-i \alpha e^{\frac{\mu}{x}-\sigma x}\right]$ (9) Inserting the above expansion in (6) gives

$$
\begin{aligned}
f(x)=\alpha & \alpha \sum_{i=0}^{\infty} \frac{(-1)^{i}}{i !} \frac{\Gamma(\beta)}{\Gamma(\beta-i)}\left\{\frac{\mu}{x^{2}}+\sigma\right\} e^{\frac{\mu}{x}-\sigma x} \\
& \times \exp \left[-(i+1) \alpha e^{\frac{\mu}{x}-\sigma x}\right]
\end{aligned}
$$

By using the power series for the exponential function, it can be written as follow:

$$
\exp \left[-(i+1) \alpha e^{\frac{\mu}{x}-\sigma x}\right]=\sum_{i=0}^{\infty} \frac{(-1)^{j}(i+1)^{j} \alpha^{j}}{j !} e^{\mathrm{j}\left(\frac{\mu}{x}-\sigma x\right)}
$$$$
\text { Inserting (11) in (10) gives }
$$

$$
\begin{aligned}
f(x)=\alpha \beta & \sum_{i=0}^{\infty} \sum_{j=0}^{\infty} \frac{(-1)^{i+j}}{i ! j !} \frac{(i+1)^{j} \alpha^{j} \Gamma(\beta)}{\Gamma(\beta-i)} \\
& \times\left\{\mu x^{-2}+\sigma\right\} e^{(\mathrm{j}+1) \frac{\mu}{x}} e^{-(\mathrm{j}+1) \sigma x}
\end{aligned}
$$

Finally, by using the series expansion

$$
e^{(\mathrm{j}+1) \frac{\mu}{x}}=\sum_{k=0}^{\infty} \frac{(j+1)^{k} \mu^{k}}{k !} x^{-k}
$$

The PDF of KIFWE distribution can be expressed as

$$
\begin{aligned}
f(x)= & \alpha \beta \sum_{i=}^{\infty} \sum_{j=0}^{\infty} \sum_{k=0}^{\infty} \frac{(-1)^{i+j}}{i ! j ! k !} \frac{(i+1)^{j} \alpha^{j}(\mathrm{j}+1)^{k} \mu^{k} \Gamma(\beta)}{\Gamma(\beta-i)} \\
& \times\left\{\mu x^{-(k+2)} e^{-(\mathrm{j}+1) \sigma x}+\sigma x^{-k} e^{-(\mathrm{j}+1) \sigma x}\right\}
\end{aligned}
$$

Thus, some statistical properties of the proposed distribution can be derived from (14). For example, the moment and moment generating function of $\mathrm{X}$ can be obtained from this equation.

\subsection{Quantile and Simulation of KIFW}

The quantile of the $K I F W$ distribution is obtained by solving the following equation, with respect to $x_{q}$

$$
F(x q)=q, \quad 0<q<1
$$

Using the distribution function of $K I F W$ distribution, from (6), we have

$$
1-\left[1-\mathrm{e}^{-\alpha e^{\frac{\mu}{x_{q}}-\sigma x_{q}}}\right]^{\beta}=q
$$

By solving the above equation, we obtain

$$
\sigma x_{q}^{2}+c(q) x_{q}-\mu=0
$$

Where

$$
c(q)=\log _{e}\left\{-\frac{\log _{e}\left(1-[1-q]^{\frac{1}{\beta}}\right)}{\alpha}\right\}
$$

By solving the above equation, we obtain $x_{q}$ as follows

$$
x_{q}=\frac{-c(q) \mp \sqrt{c(q)^{2}+4 \sigma \mu}}{2 \sigma}
$$

Since the quantile $x_{q}$ is positive, then we obtain $x_{q}$ as follows

$$
x_{q}=\frac{-c(q) \mp \sqrt{c(q)^{2}+4 \sigma \mu}}{2 \sigma}
$$

So, the simulation of the KIFW distribution random variable is straightforward. Let $U$ be a uniform variable of the unit interval $(0,1)$. Thus by means of inverse transformation method, we consider the random variable $\mathrm{X}$ given by

$X=\frac{-c(u)+\sqrt{c(u)^{2}+4 \sigma \mu}}{2 \sigma}$

Since the median is $50 \%$ quantile, then by setting $q=\frac{1}{2}$ in equation (17), we get the median of $K I F W$ distribution.

\subsection{The Moments}

The $r$ - th moment of the random variable $X$ with probability density function $f(x)$ is given by 


$$
\mu_{r}^{\prime}=\int_{-\infty}^{\infty} x^{r} f(x) d x
$$

Substituting from Eq. (14) into (18), as follow:

$$
\begin{aligned}
& \mu_{r}^{\prime}=\alpha \beta \sum_{i=j}^{\infty} \sum_{j=0}^{\infty} \sum_{k=0}^{\infty} \frac{(-1)^{i+j}}{i ! j ! k !} \frac{(i+1)^{j} \alpha^{j}(\mathrm{j}+1)^{k} \mu^{k} \Gamma(\beta)}{\Gamma(\beta-i)} \\
& \int_{0}^{\infty}\left\{\mu x^{r-(k+2)} e^{-(\mathrm{j}+1) \sigma x}+\sigma x^{r-k} e^{-(\mathrm{j}+1) \sigma x}\right\} d x
\end{aligned}
$$

By using the definition of gamma function, in the form

$$
\Gamma(\lambda)=\int_{0}^{\infty} \eta^{\lambda} t^{\lambda-1} e^{-\eta t^{\lambda}} d t, \quad \lambda, z>0
$$

We obtain the $r-t h$ moment of KIFW distribution in the form

$$
\begin{aligned}
\mu_{r}^{\prime}= & \alpha \beta \sum_{i=0}^{\infty} \sum_{j=0}^{\infty} \sum_{k=0}^{\infty} \frac{(-1)^{i+j}}{i ! j ! k !} \frac{(i+1)^{j} \alpha^{j}(\mathrm{j}+1)^{k} \mu^{k} \Gamma(\beta)}{\Gamma(\beta-i)} \\
& \left\{\frac{\mu \Gamma(r-(k+1)}{[(j+1) \sigma]^{r-(k+1)}}+\frac{\sigma \Gamma(r-(k-1)}{[(j+1) \sigma]^{r-(k-1)}}\right\}
\end{aligned}
$$

\subsection{The Moment Generating Function}

The moment generating function of the random variable $X$ with the density function $f(x)$ is given by

$$
M_{X}(t)=\int_{-\infty}^{\infty} e^{t x} f(x) d x
$$

Using series expansion of $e^{t x}$, we obtain

$$
M_{X}(t)=\sum_{r=0}^{\infty} \frac{t^{r}}{r !} \mu_{r}^{\prime}
$$

Substituting from Eq. (20) into Eq. (22) we obtain the moment generating function of $K I F W$ distribution in the form

$$
\begin{gathered}
M_{X}(t) \sum_{i=0}^{\infty} \sum_{j=0}^{\infty} \sum_{k=0}^{\infty} \sum_{r=0}^{\infty} \frac{(-1)^{i+j}}{i ! j ! k ! r !} \frac{(i+1)^{j} \alpha^{j}(\mathrm{j}+1)^{k} \mu^{k} \Gamma(\beta) t^{r}}{\Gamma(\beta-i)} \\
\quad \times\left\{\frac{\mu \Gamma(r-(k+1)}{[(j+1) \sigma]^{r-(k+1)}}\right. \\
\left.+\frac{\sigma \Gamma(r-(k-1)}{[(j+1) \sigma]^{r-(k-1)}}\right\}
\end{gathered}
$$

\section{PARAMETER ESTIMATION}

In this section, the maximum likelihood estimation is used to estimate the unknown parameters. Let $X_{1}, X_{2}, \ldots, X_{n}$ be a random sample of size $n$ from the KIFW distribution given by (6). The likelihood function for the vector of parameters $\boldsymbol{\Theta}=(\alpha, \beta, \mu, \sigma)$ can be expressed as

$$
\begin{aligned}
l f=\alpha^{n} \beta^{n} & \prod_{i=1}^{n}\left\{\frac{\mu}{x_{i}^{2}}+\sigma\right\} e^{\sum_{i=1}^{n}\left(\frac{\mu}{x_{i}}-\sigma x_{i}\right)} e^{-\alpha \sum_{i=1}^{n} e^{\frac{\mu}{x_{i}}-\sigma x_{i}}} \\
& \prod_{i=\mathbf{1}}^{n}\left\{\mathbf{1}-\boldsymbol{e}^{\sum_{i=1}^{n}\left(\frac{\boldsymbol{\mu}}{\boldsymbol{x}_{\boldsymbol{i}}}-\boldsymbol{\sigma} x_{i}\right)}\right\}^{\boldsymbol{\beta}-\mathbf{1}}
\end{aligned}
$$

Hence, the log-likelihood function $\ell(\boldsymbol{\Theta})$ becomes

$$
\begin{aligned}
\ell(\boldsymbol{\Theta})= & n \cdot\left[\log _{e}(\alpha)+\log _{e}(\beta)\right]+\sum_{i=1}^{n} \log _{e}\left(\frac{\mu}{x_{i}^{2}}+\sigma\right) \\
& +\sum_{i=1}^{n}\left(\frac{\mu}{x_{i}}-\sigma x_{i}\right)-\alpha \sum_{i=1}^{n} \frac{\mu}{x_{i}}-\sigma x_{i}
\end{aligned}
$$

$$
+(\beta-1) \sum_{i=1}^{n} \log _{e}\left(1-e^{-\alpha e^{\frac{\mu}{x_{i}}-\sigma x_{i}}}\right)
$$

The partial derivatives of $\ell(\Theta)$ with respect to each parameter $\alpha, \beta, \mu$ and $\sigma$ are given by:

$$
\begin{gathered}
U_{\alpha}=\frac{n}{\alpha}-\sum_{i=1}^{n} e^{\frac{\mu}{x_{i}}-\sigma x_{i}}+(\beta-1) \sum_{i=1}^{n} \frac{e^{\frac{\mu}{x_{i}}-\sigma x_{i}} e^{-\alpha e^{\frac{\mu}{x_{i}}-\sigma x_{i}}}}{\left(1-e^{-\alpha e^{\frac{\mu}{x_{i}}-\sigma x_{i}}}\right)} \\
U_{\beta}=\frac{n}{\beta}+\sum_{i=1}^{n} \log _{e}\left(1-e^{-\alpha e^{\frac{\mu}{x_{i}}-\sigma x_{i}}}\right) \\
U_{\mu}=\sum_{i=1}^{n} \frac{1}{x_{i}^{2}\left(\frac{\mu}{x_{i}^{2}}+\sigma\right)}+\sum_{i=1}^{n} \frac{1}{x_{i}}-\alpha \sum_{i=1}^{n} \frac{e^{\frac{\mu}{x_{i}}-\sigma x_{i}}}{x_{i}}+ \\
\alpha(\beta-1) \sum_{i=1}^{n} \frac{e^{\frac{\mu}{x_{i}}-\sigma x_{i}} e^{-\alpha e^{\frac{\mu}{x_{i}} \sigma x_{i}}}}{x_{i}\left(1-e^{-\alpha e^{\frac{\mu}{x_{i}}-\sigma x_{i}}}\right)} \\
U_{\sigma}=\sum_{i=1}^{n} \frac{1}{\left(\frac{\mu}{x_{i}^{2}}+\sigma\right)}-\sum_{i=1}^{n} x_{i}+\alpha \sum_{i=1}^{n} x_{i} e^{\frac{\mu}{x_{i}}-\sigma x_{i}} \\
-\alpha(\beta-1) \sum_{i=1}^{n} \frac{x_{i} e^{\frac{\mu}{x_{i}}-\sigma x_{i}} e^{-\alpha e^{\frac{\mu}{x_{i}}}-\sigma x_{i}}}{\left(1-e^{-\alpha e^{\frac{\mu}{x_{i}}-\sigma x_{i}}}\right)}
\end{gathered}
$$

Setting these equations to zero and solving them simultaneously yields the MLEs of the four parameters. For interval estimation of the model parameters, we require the $4 \times 4$ observed information matrix $J(\Theta)=\left\{U_{r s}\right\}$ (for $r, s=$ $\alpha, \beta, \mu, \sigma)$ given in Appendix. Under standard regularity conditions, the multivariate normal $N_{4}\left(0 ; J(\widehat{\Theta})^{-1}\right)$ distribution can be used to construct approximate confidence intervals for the model parameters. (Cox and Hinkley [17]), here, $J(\widehat{\Theta})$ is the total observed information matrix evaluated at $\widehat{\Theta}$. Then, the $100(1-\gamma) \%$ confidence intervals for $\alpha, \beta, \mu$ and $\sigma$ are given by:

$\hat{\alpha}+z_{\frac{\gamma}{2}} \sqrt{\operatorname{var}(\hat{\alpha})}, \hat{\beta}+z_{\frac{\gamma}{2}} \sqrt{\operatorname{var}(\hat{\beta})}, \hat{\mu}+z_{\frac{\gamma}{2}} \sqrt{\operatorname{var}(\hat{\mu})}$ and $\hat{\sigma}+$ $Z_{\frac{\gamma}{2}} \sqrt{\operatorname{var}(\hat{\sigma})}$ respectively, where the $\operatorname{var}($.$) 's denote the$ diagonal elements of $J(\widehat{\Theta})^{-1}$ corresponding to the model parameters, and $Z_{\frac{\gamma}{2}}$ is the quantile $\left(1-\frac{\gamma}{2}\right) 100 \%$ of the standard normal distribution. The likelihood ratio LRT statistic can be used to check if the KIFW distribution is strictly "superior" to the $I F W$ distribution for a given data set. Then, the test of $H_{0}: \alpha=\beta=1$ versus $H_{1}: \alpha \neq \beta \neq 1$ is equivalent to compare the $K I F W$ and $I F W$ distributions and the LRT statistic becomes $\Lambda=2\{\ell(\hat{\alpha}, \hat{\beta}, \hat{\mu}, \hat{\sigma})-\ell(1,1, \tilde{\mu}, \tilde{\sigma})\}$, where $\hat{\alpha}, \hat{\beta}, \hat{\mu}$ and $\hat{\sigma}$ are the MLEs under $H_{1}$ and $\tilde{\mu}$ and $\tilde{\sigma}$ are the estimates under $H_{0}$. The LRT statistic $\Lambda$ is asymptotically (as $n \rightarrow \infty$ ) distributed as $\chi_{q}^{2}$, where $q$ is the number of parameters specified under $H_{0}$. The LRT rejects $H_{0}$ if $\Lambda>\chi_{q ; 1-\gamma}^{2}$, where $\chi_{q ; 1-\gamma}^{2}$ denotes the $100 \gamma \%$ quantile of the $\chi_{q}^{2}$ distribution.

\section{SIMULATION RESULTS}

We shall report the results from a Monte Carlo experiment on the finite sample behavior of the MLEs of the parameters $\alpha, \beta, \mu$ and $\sigma$. The simulation was carried out using the $\mathrm{GW}$ Basic programming language and were obtained from 1,000 Monte Carlo replications. In each replication, a random sample of size $\mathrm{n}$ is drawn from the $\operatorname{KIFW}(\alpha, \beta, \mu, \sigma)$ distribution and the parameters were estimated by maximum 
likelihood. In Table 1, we present the means of the MLEs of the four parameters with the corresponding bias and root mean squared error (RMSE) for sample sizes 50, 100 and 200. The true parameters values used in the data generating processes are $\alpha=1.5, \beta=0.5, \mu=2.0$ and $\sigma=1.5$. Based on the figures in Table 1, we note that the MSEs of the estimates decay toward zero as the sample size increases, as usually expected under standard regularity conditions. As the sample size $\mathrm{n}$ increases, the mean estimates of the parameters tend to be closer to the true parameter values. This fact supports that the asymptotic normal distribution provides an adequate approximation to the finite sample distribution of the estimates.

Table 1. Mean estimates and root mean squared errors of the MLEs based Monte Carlo simulation.

\begin{tabular}{|c|c|c|c|c|c|}
\hline \multicolumn{2}{|c|}{ Parameters } & $\alpha$ & $\beta$ & $\mu$ & $\sigma$ \\
\hline \multirow{3}{*}{ 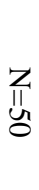 } & Mean & 1.50018 & 0.51999 & 2.05879 & 1.50081 \\
\hline & Bias & 0.00018 & 0.01999 & 0.05879 & 0.00081 \\
\hline & RMSE & 0.00307 & 0.08050 & 0.22873 & 0.02196 \\
\hline \multirow{3}{*}{$\frac{7}{\frac{Z}{8}}$} & Mean & 1.50000 & 0.50909 & 2.02991 & 1.50003 \\
\hline & Bias & 0.00 & 0.00909 & 991 & 0.00003 \\
\hline & RMs & 0.00019 & 0.05662 & 0.15828 & 0.00019 \\
\hline \multirow{3}{*}{$\begin{array}{l}\text { ZI } \\
\text { ㅁ } \\
\stackrel{8}{0}\end{array}$} & Mean & 1.50000 & 0.50570 & 2.01643 & 1.50001 \\
\hline & Bias & 0.00000 & 0.00570 & 0.01643 & 0.00001 \\
\hline & RMSE & 0.00003 & 0.03685 & 0.10910 & 0.00003 \\
\hline
\end{tabular}

\section{APPLICATIONS}

In this section, we illustrate the usefulness of the KIFW distribution. We fit this distribution to the real data sets and compare the results with the Inverse Weibull $I W$ and Inverse flexible Weibull Extension IFW distributions. The data set given in Table 2 is taken from Murthy, Xie, and Jiang (2004) page 180 [18] and represents 50 items put into use at $t=0$ and failure times are in weeks.

Table 2: 50 items put into use at $t=0$ and their failure times in weeks

\begin{tabular}{|l|l|l|l|l|l|}
\hline 0.013 & 0.065 & 0.111 & 0.111 & 0.163 & 0.309 \\
\hline 0.426 & 0.535 & 0.684 & 0.747 & 0.997 & 1.284 \\
\hline 1.304 & 1.647 & 1.829 & 2.336 & 2.838 & 3.269 \\
\hline 3.973 & 3.981 & 4.520 & 4.789 & 4.849 & 5.202 \\
\hline 5.291 & 5.349 & 5.911 & 6.018 & 6.427 & 6.456 \\
\hline 6.572 & 7.023 & 7.087 & 7.291 & 7.787 & 8.596 \\
\hline 9.388 & 10.281 & 10.713 & 11.658 & 13.006 & 13.388 \\
\hline 13.842 & 17.152 & 17.283 & 19.418 & 23.471 & 24.777 \\
\hline 32.795 & 48.105 & & & & \\
\hline
\end{tabular}

Tables 3 provide the MLEs of the model parameters. The model selection is carried out using the AIC (Akaike information criterion), the BIC (Bayesian information criterion) and the CAIC (Consistent Akaike information criteria):

$A I C=2 q-2 \ell(\widehat{\Theta}), \quad B I C=q \log (n)-2 \ell(\widehat{\Theta})$ and $C A I C=$ $\frac{2 q n}{n-q-1}-2 \ell(\widehat{\boldsymbol{\Theta}})$. Where $\ell(\widehat{\boldsymbol{\Theta}})$ denotes the log-likelihood function evaluated at the maximum likelihood estimates, $q$ is the number of parameters and $\mathrm{n}$ is the sample size. Since the values of the AIC, BIC and CAIC are smaller for the KIFW distribution compared with those values of the other models, the new distribution seems to be a very competitive model to these data.

Table 3: MLEs of the model parameters, the corresponding SEs (given in parentheses) and the statistics AIC, BIC and CAIC Estimates Statistic

\begin{tabular}{|c|c|c|c|}
\hline Model & IW & IFW & KIFW \\
\hline $\mathrm{a}$ & & & $\begin{array}{c}1.819 \\
(0.00069)\end{array}$ \\
\hline $\mathrm{b}$ & & $\begin{array}{c}0.854 \\
(0.12029)\end{array}$ \\
\hline$\mu$ & $\begin{array}{c}1.125 \\
(0.16557)\end{array}$ & $\begin{array}{c}0.027 \\
(0.00424)\end{array}$ & $\begin{array}{c}0.015 \\
(0.00429)\end{array}$ \\
\hline$\sigma$ & $\begin{array}{c}0.479 \\
(0.04542\end{array}$ & $\begin{array}{c}0.136 \\
(0.01878)\end{array}$ & $\begin{array}{c}0.181 \\
(0.00011)\end{array}$ \\
\hline$\ell(\widehat{\boldsymbol{\theta}})$ & -168.641 & -161.070 & -153.334 \\
\hline AIC & 341.282 & 326.140 & 314.668 \\
\hline BIC & 345.106 & 329.964 & 322.316 \\
\hline CAIC & 341.537 & 326.395 & 315.557 \\
\hline
\end{tabular}

The $I F W$ distribution is a special case of $K I F W$ distribution. We want to test if these data fit the $I F W$ or the $K I F W$, using the likelihood ratio test $\Lambda=2\{\ell(\hat{\alpha}, \hat{\beta}, \hat{\mu}, \hat{\sigma})-\ell(1,1, \tilde{\mu}, \tilde{\sigma})\}$. The hypotheses are as follows:

$$
\begin{gathered}
\qquad H_{0}: \alpha=1, \beta=1(I F W) \\
\text { versus } H_{1}: \alpha \neq 1, \beta \neq 1(K I F W) .
\end{gathered}
$$

The likelihood ratio statistic $\Lambda$ is 15.472 . We observed that the calculated LRT statistic is greater than the upper $99 \%$ quantile of a chi-square random variable with 2 degree of freedom $\chi_{2,99 \%}^{2}=9.210$, it is clear that we reject the null hypotheses. We can therefore conclude that this data fits the KIFW distribution much better than the IFW distribution. 


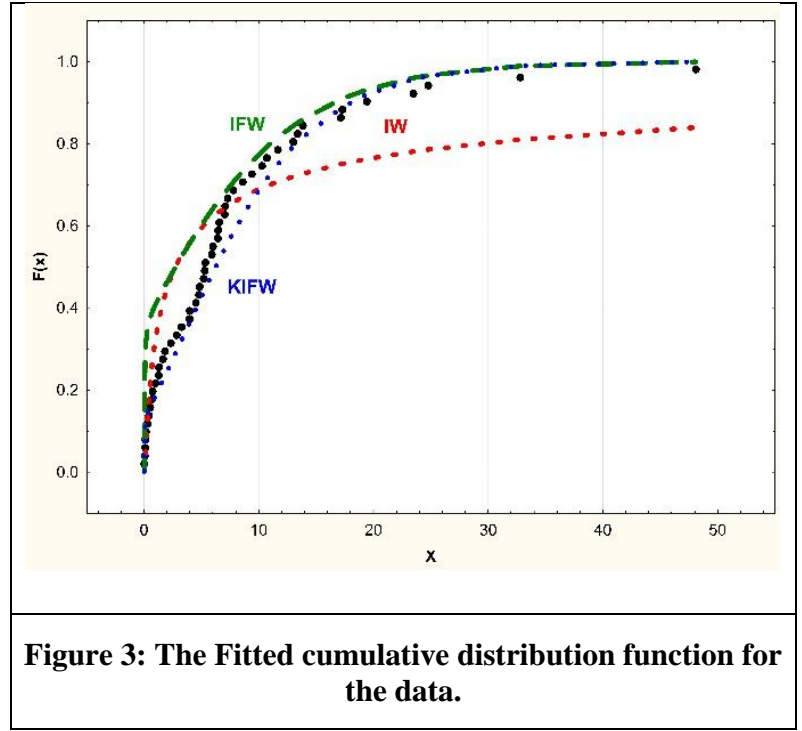

\section{CONCLUSIONS}

Here is a new proposal model that concluded from the current essay and it's called kumaraswamy inverse flexible Weibull extension distribution which extends the inverse flexible Weibull extension distribution in the analysis of data with real support. An obvious reason for generalizing a standard distribution is because the generalized form provides more flexibility in modeling real data. We derive expansions for moments and for the moment generating function. The estimation of parameters is approached by the method of maximum likelihood, also the information matrix is derived. An application of the KIFW distribution to real data show that the new distribution can be used quite effectively to provide better fits than the IFW distribution.Finally, we hope that the proposed model will attact wider applications in reliability engineering, survival and life time data.

\section{REFERENCES}

[1] El-Gohary, A., El-Bassiouny, A. H. and El-Morshedy M. (2015). Inverse Flexible Weibull Extension Distribution, International Journal of Computer Applications, 115(2), 46-51.

[2] Bebbington, M. S., Lai, C. D. and Zitikis, R. (2007). A flexible Weibull extension. Reliability Engineering \& System Safety, 92(6), 719-26.

[3] Marshall A.W. and Olkin I. (1997). A new method for adding a parameter to a family of distributions with application to the exponential and Weibull families, Biometrika, 84, 641-652.

[4] Eugene, N., et al. (2002). Beta-Normal distribution and its applications. Communications in Statistics - Theory and Methods, 31(4), 497-512.

[5] Shaw. W.T, Buckley I.R.C., (2007). The alchemy of probability distributions: beyond Gram- Charlier expansions, and a skew-kurtotic-normal distribution from a rank transmutation map. Technical report.

[6] Cordeiro G.M. and de Castro M. (2011). A new family of generalized distributions. Journal of Statistical Computation and Simulation, 81(7), 883-898.

[7] Alexander, C., Cordeiro, G.M., Ortega, E.M.M. and Sarabia, J.M. (2012). Generalized beta generated distributions, Computational Statistics and Data Analysis, 56, 1880-1897.

[8] Zografos, K. and Balakrishnan, N. (2009). On families of beta- and generalized gamma-generated distributions and associated inference. Statistical Methodology, 6, 344362.

[9] Ristic M.M., Balakrishnan N. (2012). The Gammaexponentiated Exponential Distribution. Journal of Statistical Computation and Simulation, 82(8), 11911206.

[10] Amini, M., Mir Mostafaee, S.M.T.K. and Ahmadi, J. (2012). Log-gamma-generated families of distributions, Statistics, 48, 913-932.

[11] Torabi, H. and Montazari, N.H. (2014). The logisticuniform distribution and its application. Communications in Statistics-Simulation and Computation, 43, 25512569.

[12] Cordeiro, G.M., Ortega, E.M.M. and da Cunha, D.C.C. (2013). The exponentiated generalized class of distributions, Journal of Data Science, 11, 1-27.

[13] Alzaatreh, A., Lee, C. and Famoye, F. (2013). A new method for generating families of continuous distributions, Metron,

[14] Bourguignon, M., Silva, R.B. and Cordeiro, G.M. (2014). The Weibull-G family of probability distributions, Journal of Data Science, 12, 53-68.

[15] Tahir M.H., Cordeiro G.M., Alzaatreh A., Mansoor M., Zubair M. (2016).The logistic-X family of distributions and its applications, Commun. Stat. Theory Methods, (forthcoming).

[16] Jones, M.C. (2009). Kumaraswamy's distribution: A beta-type distribution with some tractability advantages. Statistical Methodology, 6, 70-81.

[17] Cox, D.R. and Hinkley, D.V. (1974). Theoretical Statistics. Chapman \& Hall, London.

[18] Murthy, D.N.P, Xie, M. and Jian, R. (2004). Weibull Models.Wiley.

\section{APPENDIX}

The elements of the observed Fisher information matrix $J(\boldsymbol{\theta})$ for the parameters $(\alpha, \beta, \mu, \sigma)$

$$
J(\boldsymbol{\theta})=-\left\{\begin{array}{cccc}
U_{\alpha \alpha} & U_{\alpha \beta} & U_{\alpha \mu} & U_{\alpha \sigma} \\
\cdot & U_{\beta \beta} & U_{\beta \mu} & U_{\beta \sigma} \\
\cdot & \cdot & U_{\mu \mu} & U_{\mu \sigma} \\
\cdot & \cdot & \cdot & U_{\sigma \sigma}
\end{array}\right\}
$$

are given by

$$
\begin{aligned}
& U_{\alpha \alpha}=-\frac{n}{\alpha^{2}}-(\beta-1) \sum_{i=1}^{n} \frac{\left(e^{\frac{\mu}{x_{i}}-\sigma x_{i}}\right)^{2} e^{-\alpha e^{\frac{\mu}{x_{i}} \sigma x_{i}}}}{\left(1-e^{-\alpha e^{\frac{\mu}{x_{i}} \sigma x_{i}}}\right)} \\
& -(\beta-1) \sum_{i=1}^{n} \frac{\left(e^{\frac{\mu}{x_{i}}-\sigma x_{i}}\right)^{2}\left(e^{-\alpha e^{\frac{\mu}{x_{i}} \sigma x_{i}}}\right)^{2}}{\left(1-e^{-\alpha e^{\frac{\mu}{x_{i}}-\sigma x_{i}}}\right)^{2}}
\end{aligned}
$$


International Journal of Computer Applications (0975 - 8887)

Volume 154 - No.7, November 2016

$$
\begin{aligned}
& U_{\alpha \beta}=\sum_{i=1}^{n} \frac{\left(e^{\frac{\mu}{x_{i}}-\sigma x_{i}}\right) e^{-\alpha e^{\frac{\mu}{x_{i}} \sigma x_{i}}}}{\left(1-e^{-\alpha e^{\frac{\mu}{x_{i}}} \sigma x_{i}}\right)} \\
& U_{\alpha \mu}=-\sum_{i=1}^{n} \frac{e^{\frac{\mu}{x_{i}}-\sigma x_{i}}}{x_{i}}+(\beta-1) \sum_{i=1}^{n} \frac{e^{\frac{\mu}{x_{i}}-\sigma x_{i}} e^{-\alpha e^{\frac{\mu}{x_{i}}-\sigma x_{i}}}}{x_{i}\left(1-e^{-\alpha e^{\frac{\mu}{x_{i}}-\sigma x_{i}}}\right)} \\
& -\alpha(\beta-1) \sum_{i=1}^{n} \frac{\left(e^{\frac{\mu}{x_{i}}-\sigma x_{i}}\right)^{2} e^{-\alpha e^{\frac{\mu}{x_{i}}-\sigma x_{i}}}}{x_{i}\left(1-e^{-\alpha e^{\frac{\mu}{x_{i}}}-\sigma x_{i}}\right)} \\
& +\alpha(\beta-1) \sum_{i=1}^{n} \frac{\left(e^{\frac{\mu}{x_{i}}-\sigma x_{i}}\right)^{2}\left(e^{-\alpha e^{\frac{\mu}{x_{i}}-\sigma x_{i}}}\right)^{2}}{x_{i}\left(1-e^{-\alpha e^{\frac{\mu}{x_{i}}-\sigma x_{i}}}\right)^{2}} \\
& U_{\alpha \sigma}=\sum_{i=1}^{n} x_{i} e^{\frac{\mu}{x_{i}}-\sigma x_{i}}-(\beta-1) \sum_{i=1}^{n} \frac{x_{i} e^{\frac{\mu}{x_{i}}-\sigma x_{i}} e^{-\alpha e^{\frac{\mu}{x_{i}}-\sigma x_{i}}}}{x_{i}\left(1-e^{-\alpha e^{\frac{\mu}{x_{i}}-\sigma x_{i}}}\right)} \\
& +\alpha(\beta-1) \sum_{i=1}^{n} \frac{x_{i}\left(e^{\frac{\mu}{x_{i}}-\sigma x_{i}}\right)^{2} e^{-\alpha e^{\frac{\mu}{x_{i}}}-\sigma x_{i}}}{\left(1-e^{-\alpha e^{\frac{\mu}{x_{i}}-\sigma x_{i}}}\right)} \\
& +\alpha(\beta-1) \sum_{i=1}^{n} \frac{x_{i}\left(e^{\frac{\mu}{x_{i}}-\sigma x_{i}}\right)^{2}\left(e^{-\alpha e^{\frac{\mu}{x_{i}}}-\sigma x_{i}}\right)^{2}}{\left(1-e^{-\alpha e^{\frac{\mu}{x_{i}}-\sigma x_{i}}}\right)^{2}} \\
& U_{\beta \beta}=-\frac{n}{\beta^{2}} \\
& U_{\beta \mu}=\alpha \sum_{i=1}^{n} \frac{e^{\frac{\mu}{x_{i}}-\sigma x_{i}} e^{-\alpha e^{\frac{\mu}{x_{i}}-\sigma x_{i}}}}{x_{i}\left(1-e^{-\alpha e^{\frac{\mu}{x_{i}}-\sigma x_{i}}}\right)} \\
& U_{\beta \sigma}=-\alpha \sum_{i=1}^{n} \frac{x_{i} e^{\frac{\mu}{x_{i}}-\sigma x_{i}} e^{-\alpha e^{\frac{\mu}{x_{i}}-\sigma x_{i}}}}{\left(1-e^{-\alpha e^{\frac{\mu}{x_{i}}-\sigma x_{i}}}\right)} \\
& U_{\mu \mu}=-\sum_{i=1}^{n} \frac{1}{x_{i}^{4}\left(\frac{\mu}{x_{i}^{2}}+\sigma\right)^{2}}-\alpha \sum_{i=1}^{n} \frac{e^{\frac{\mu}{x_{i}}-\sigma x_{i}}}{x_{i}^{2}} \\
& +\alpha(\beta-1) \sum_{i=1}^{n} \frac{e^{\frac{\mu}{x_{i}}-\sigma x_{i}} e^{-\alpha e^{\frac{\mu}{x_{i}}-\sigma x_{i}}}}{x_{i}^{2}\left(1-e^{-\alpha e^{\frac{\mu}{x_{i}}-\sigma x_{i}}}\right)}
\end{aligned}
$$

IJCATM : www.ijcaonline.org

46 